\title{
Application of an Integrated Risk Assessment Software to Quantify the Life Safety Risk in Building during a Fire
}

\author{
Adam Krasuski ${ }^{1, *}$ and Mariusz Pecio $^{1}$ \\ ${ }^{1}$ The Main School of Fire Service, Faculty of Fire Safety Engineering, 52/54 Słowackiego St., 01-629 \\ Warsaw, Poland
}

\begin{abstract}
.
We develop an integrated probabilistic risk assessment software called Aamks for the purpose of quantifying the life safety level of people present in buildings in the context of fire safety design. The software implements easy to use and computationally efficient quantitative risk assessment method - multisimulation. The method consists in deterministic sub-models and probabilistic techniques for sampling and quantifying the fire safety level by means of failure probabilities, individual and societal risk. Following the theoretical description of the method, a case study of a five storey hotel building $20.000 \mathrm{~m}^{2}$ is elaborated and discussed as proof of concept.
\end{abstract}

\section{Introduction}

One of the four key competences of a fire protection expert is the ability to prepare expert opinions and other technical studies in the area of fire protection. This is very often associated with the necessity of selecting a set of appropriate technical solutions to ensure the protection of life, health and property. An expert opinion is especially required when there is a need to choose from a range of available options, such as adjusting a building design to meet the provisions of applicable law or suggesting alternative solutions, to guarantee safety while also remaining acceptable to the investor in its financial impact. The choice of an alternative pathway involves the need to prove that the suggested concept ensures at least the level of safety required by law. In such cases, it is useful to have the tools that enable a comparison of individual variants with regard to safety. This subsequently allows the comparison of the obtained values with the legal requirements, and the selection of a variant that is acceptable to both the fire service and the investor. In order to be able to carry out such an analysis, it is necessary to transfer all the considered variants to one denominator (dimension, feature) to enable clear comparisons to be made. This denominator can be the same risk that is successfully used in other safety-related areas. Moreover, if we apply a quantitative risk analysis, it allows us to easily compare individual variants using numerical values. Quantitative risk analysis is a systematic and comprehensive methodology of risk assessment, related to technologically complex processes or objects. Due to its advantages and safety assessment capabilities, it has been successfully used in the nuclear energy and aviation industries for many years. There have been some attempts to apply it in fire protection although, due to the complicated analytical process as well as the lack of tools supporting this process, it has been

\footnotetext{
*e-mail: akrasuski@sgsp.edu.pl
} 
less than successful. However, the recent progress in the field of mathematical analyses, supported by appropriate algorithms and the computing power of computers, has resulted in the emergence of solutions enabling quantitative risk analyses to be made in a simple and precise manner. One such solution is multisimulation, allowing the implementation of quantitative risk analysis using models of fire development and evacuation as well as other models, based on the Monte Carlo method.

\section{Methods and Materials}

Multisimulation is a tool that allows the implementation of quantitative risk analysis, in particular to justify the adopted solutions in relation to any alternative solutions for existing buildings or deviations from the legal provisions for newly designed buildings. The primary concept behind multisimulation is to execute numerous computer simulations (hence the name) for the largest possible number of fire scenarios in a given building, and then to determine the probability distributions for individual scenarios and their consequences [1]. The analysis of all possible scenarios under multisimulations is possible thanks to the use of the Monte Carlo method [2]. This method generates uncertain input parameters for the fire and evacuation models embedded in the multisimulations (Fig. 1). The input parameters include the strength of the fire, the speed of fire development, the condition of the fire protection devices, the venting conditions in the building, the site of the fire, people's reaction, fire safety systems, etc. This makes multisimulation significantly differs from the direct use of CFD in a limited number of scenarios. Multisimulation is well grounded in both scientific and normative studies. The basis for this method include NFPA 551 [3], NFPA 101 [4], ISO/TS 16733 [5] and the technical principles described by the SFPE Engineering Guide [6] or SFPE Handbook of Fire Protection Engineering [7]. In most cases, a comparative analysis is used in accordance with the method set out in British Standard PD 7974 [8]. The input parameters of the multisimulation is determined on the basis of an SWD-ST database analysis and numerous published experiment results.

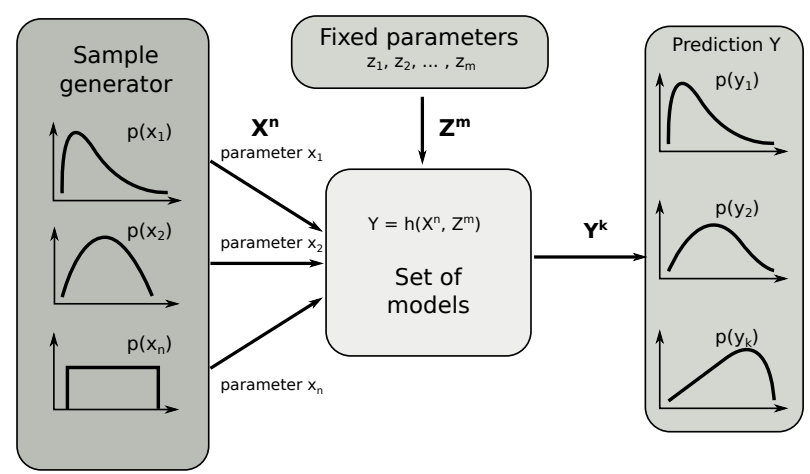

Figure 1. The construction principle of the multisimulation. $X^{n}$ is a vector length $n$ of independent copies of random variable $\mathrm{X}$ providing the input for the models, $Z^{m}$ is a vector length $m$ of fixed parameters providing the input for the models, $Y^{k}$ is a vector length $k$ predicted output parameters.

The multisimulation method requires an appropriate computer program and suitable parameter definitions for the analysed object. The available free software includes PFS [9], and B-RISK [10]. In this article we use software developed by The Main School of Fire Services 
in cooperation with the Aalto University in Finland called Aamks ${ }^{1}$. This modern and open source web-based application with the support of cloud computing, makes multisimulation feasible to apply in day-to-day building design. Aamks models fire and evacuation jointly and the consequences of fire to people are calculated taking into account smoke toxicity by means of fractional effective dose (FED). This method allows the determination of the amount of fire gases each person has inhaled and what the consequences of this are. We calculated FED, according to the formula presented in $[7,11]$.

A FED value higher than or equal to $1[7,11]$ is assumed as a lethal dose. The impacts of gas amounts leading to a FED value below 1 on the human body are presented in Table 1 .

Table 1. FED effects on human health in Aamks.

\begin{tabular}{|c|c|}
\hline FED & Effect on human health \\
\hline$<0.01$ & Minor or negligible \\
\hline$[0.01-0.3[$ & Low - short period of hospitalization \\
\hline$[0.3-1[$ & Heavy - lingering health problems or permanent disability \\
\hline$\geq 1$ & Lethal \\
\hline
\end{tabular}

The influence of temperature and thermal radiation flux is determined in the form of limit values. It is deemed that evacuation is possible if the smoke temperature does not exceed $60^{\circ} \mathrm{C}$ at a height of $1.8 \mathrm{~m}$, and the radiation flux does not exceed $2.5 \mathrm{~kW} / \mathrm{m}^{2}$ [7]. The heat radiation flux criterion is sometimes expressed alternatively as the temperature of the smoke layer at the ceiling level not exceeding $200^{\circ} \mathrm{C}$, which corresponds to a radiation of approximately $2.5 \mathrm{~kW} / \mathrm{m}^{2}$. The above methodology, in combination with the multisimulation approach, allows the determination of the probability and consequences of individual scenarios, which enables the implementation of a full quantitative risk analysis.

In order to better approximate the functioning and use of the multisimulation method and Aamks software, we have used a practical example. The case under consideration is the change in use of a building. This building is a medium rise office building of category ZL III, with five overground storeys.

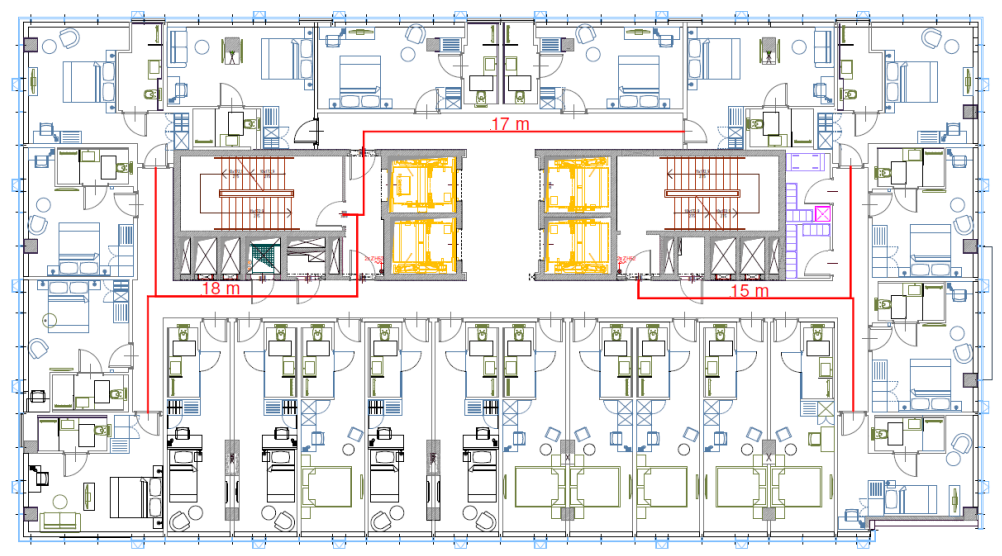

Figure 2. The designed space arrangement after the category change

\footnotetext{
${ }^{1}$ github.com/aamks
} 
As part of the investment process, the building is to be transformed into a hotel (ZL V) with the same arrangement of rooms on each floor. Figure 2 presents the arrangement for each floor after the transformation. As a result of changing the hazard category for people, a number of inconsistencies have arisen which must be rectified in order for the building to receive authorisation for its change of use. These inconsistencies are: a) The length of an emergency route in one evacuation direction has been exceeded. The length required for ZL III is $20 \mathrm{~m}$, but category ZL V demands a new maximum length of $10 \mathrm{~m}$. Figure 2 shows the escape routes where these requirements are not met. b) No fire alarm system (SSP), which is required for a hotel with a capacity exceeding 50 beds. c) There are no doors of fire resistance class EI 30 in the hotel rooms.

In order to obtain authorisation for the change of use and category, there are two main ways of dealing with the required fire safety concept: direct adaptation to the requirements of the regulations and alternative solutions. Within each of these pathways, various solutions can be applied. In order to illustrate the process of developing a solution, let us assume that an expert considers the following variants.

Variant 1.1 The proposed variant assumes adjusting the room arrangement directly to meet the requirements of the regulations. This is achieved by dividing each floor into rooms in such a way that the length of the emergency route in any evacuation direction does not exceed $10 \mathrm{~m}$. In addition, SSP and EI 30 doors must be installed to meet the other requirements. In order to ensure the maximum length of the emergency route does not exceed $10 \mathrm{~m}$, it is necessary to combine several rooms into one. With the arrangement method suggested by this variant, the number of rooms is reduced by 7 for each floors.

Variant 1.2 The proposed variant assumes adjusting the room arrangement directly to meet the requirements of the regulations. Acceptable emergency route lengths without changing the interior arrangement can be achieved using a sprinkler system, to allow the route length to be extended by $50 \%$, and a smoke exhaust ventilation system, to allow the evacuation route length to be extended by a further $50 \%$. This gives a total of $20 \mathrm{~m}$. In this configuration, none of the routes in the system exceed this value. Other inconsistencies can also be removed thanks to the use of SSP and EI 30 doors.

Variant 2.1 The scope of this option involves an alternative solution. The safety concept here is the use of smoke ventilation, which will extend the length of the emergency routes by $50 \%$, i.e. to $15 \mathrm{~m}$, and of additionally illuminated emergency exit signs with a light intensity of 5 lux. It is assumed that the lacking 3 metres of the emergency route length will be compensated by the use of adequate emergency lighting. Other inconsistencies can also be removed thanks to the use of SSP and EI 30 doors.

Variant 2.2 Variant 2.2 also provides an alternative solution. The safety concept here is the use of a sprinkler system, which, like the smoke ventilation system, extends the length of the emergency route by $50 \%$, i.e. to $15 \mathrm{~m}$, and of additionally illuminated evacuation signs with a light intensity of 5 lux. It is assumed that the lacking 3 metres of the emergency route length will be compensated for by adequate emergency lighting. Other inconsistencies will also be removed thanks to the use of SSP and EI 30 doors.

Variant 2.3 In this variant, the lack of some technological solutions is compensated for by the use of procedural solutions. In the terms of technical solutions, the following elements are used: a single-stage alarm system, illuminated emergency exit signs and, additionally, the separation of rooms with EI 60 partitions, including the use of fire-resistant installation culverts. In the scope of procedural solutions, the following elements are used: appropriate training for personnel, fire protection audits and the appointment of people responsible for evacuation from a given floor. It is assumed that such a combination of solutions will ensure an adequate level of fire safety. 
Variant 2.4 For this solution, as in the previous ones, the following elements are used: SSP and EI 30 doors, and, in order to compensate for the exceeded emergency route length, illuminated emergency exit signs. We assume that this solution will be equally effective.

The use of variant 1.1 is a simple and relatively cheap way of removing the inconsistencies. However, it will most probably be questioned by the investor, as decreasing the number of rooms may result in a significant reduction in the return on investment. The number of rooms is related directly to the achievable profits, thus the investor may find option 1.1 difficult to accept.

Variant 1.2 also removes all inconsistencies through the use of additional ventilation and sprinkler systems. This solution is often difficult in terms of installation, due to problems related to installing the smoke ventilation system and the required length of the ducts, which also generates high costs. The costs of sprinkler and ventilation systems are very high, and significantly extend the time needed for achieving a return on investment.

Variants 2.1 and 2.2 are cost-optimised versions of variant 1.2, consisting of replacing the sprinkler (variant 2.1) or smoke ventilation (variant 2.2) installation with other solutions. Due to the inconsistency with the requirements of the legal provisions, these variants may be possible to implement on the basis of an expert opinion. However, they are associated with the same disadvantages (to a lesser extent) as variant 1.2. This means problems related to the installation and relatively high investment costs. Moreover, projects carried out on the basis of an expert opinion are associated with an uncertainty in the final effect.

Variant 2.3 seems to be acceptable to the investor in terms of costs; however, it generates additional problems in the form of a large number of possible false alarms and the inconvenience of conducting training, reviews and audits. Moreover, due to the inconsistency with the requirements of the legal provisions, these variants may only be possible to implement on the basis of an expert opinion.

Variant 2.4 is the cheapest to implement; however, it has a very limited number of alternative solutions available. Moreover, since the project would be carried out on the basis of an expert opinion, it is associated with a great deal of uncertainty regarding the final effect.

The installation of water extinguishers or mechanical ventilation constitutes an unacceptably high cost. Therefore, suggestions are included for solutions aimed at improving the parameters of the existing fire protection devices (illuminated emergency exit signs, change of alarm and control mode, and increase in emergency lighting intensity) as well as organisational solutions, including increasing the frequency of evacuation drills, conducting quarterly fire protection inspections etc. The power of these arguments, however, may be unconvincing in relation to the existing inconsistencies (for example, the lack of smoke ventilation in the horizontal emergency routes). Developing an expert opinion for this building is a difficult task because there is a spectrum of solutions, and they are difficult to compare, such as how to compare the effectiveness of very different technologies, e.g. sprinklers with alarm procedures. Therefore, in order to obtain reliable confirmation that these solutions would achieve the achieved fire safety level indicated in the regulations, a relevant method should be used to compare the respective solutions. Undoubtedly, the best way to perform the comparison is to compare numerical values, which is possible using multisimulation.

\section{Results}

In order to compare the risk for individual variants of the fire safety concept, an average of about 3000 computer simulations were performed for each of the variants. The analysis included various fire scenarios, taking into account the different materials subject to combustion, the speed of development and the strength of the fire, the location of the fire and the reaction of the people and safety systems. As most input parameter distributions in the 
multisimulation were continuous, it should be assumed that the number of fire scenarios considered corresponded to the number of simulations performed. With such a large number of scenarios, a simplified event tree was adopted by visualising grouped scenarios. Figure 3 shows the event tree for the considered fire scenarios in the building, grouped according to the consequences of the considered scenarios. Each fire scenario was characterized by the probability $(\mathrm{P})$ of its occurrence and specific effects $(\mathrm{S})$. Losses were determined based on the development of FED values, where: H (HIGH) - death, M (MODERATE) - severe injuries, L (LOW) - light injuries and N (NEGLIGIBLE) - minor or negligible injuries.

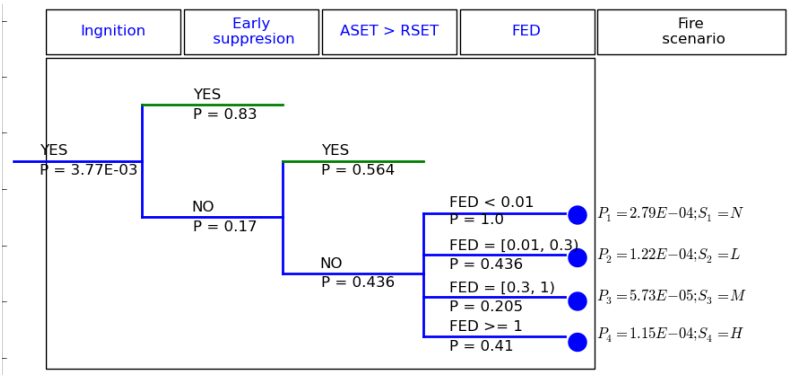

Figure 3. The exemplary event tree use as output from multisimulation

Based on the data presented in the event tree for consecutive variants, the risks for individual scenarios and their effects were calculated. The risk value was calculated as the product of the probability multiplied by the effects $[8,12,13]$ : where:

$$
R_{i}=P_{i} \times S_{i}
$$

$\mathrm{i}$ - respective risks from the event tree.

Next, on the basis of the international SFPE guidelines [7, 8], the risks were assessed by placing them in the risk matrix. The evaluation was aimed at determining the risks that were negligible, acceptable or demanded reduction. Figure 4 presents the risk matrix for the building under consideration, divided into the individual variants of the interior arrangement.

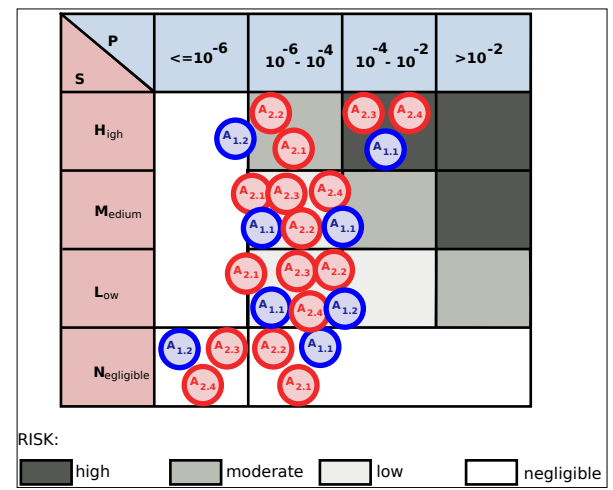

Figure 4. Ranking the risks with using SFPE Risk matrix.

Variant 1.2 was characterized by the lowest risk, categorized as negligible to moderate. The maximum risk level for variants 2.1 and 2.1 was moderate, while variants $1.1,2.3$ and 
2.4 entered the high risk area. This specification confirmed the effectiveness of the smoke ventilation and sprinkler systems in building protection, their coexistence reducing the risk to the lowest level, with installations involving only one system type maintaining the risk at the moderate level. The variants without either of these installations entered the high risk area. The probabilities for the risks presented in the risk matrices were considered to be the number of scenarios in the multisimulation in which a specific event occurred (e.g. DCBE $<\mathrm{WCBE}$ ), in relation to the number of all scenarios in the multisimulation. This defined the probability that a given scenario may occur. However, the risk matrix did not provide the opportunity to present how many people would suffer injuries in a given scenario. Breaking down the consequences within one category, e.g. HIGH, to the number of people at risk was possible by applying FN-curves. These showed the probability in the function of individual effects (number of people). In view of the fact that risk profiles presented the probability of the occurrence of victims as a function of the number of people, they were used as a method of defining societal risk. Figure 5 presents the exemplary risk profiles for deaths and severe and minor injuries.
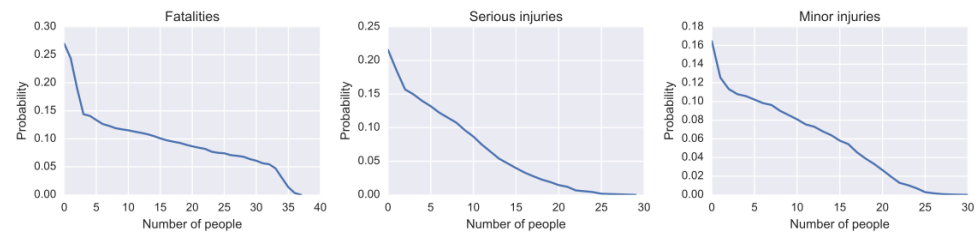

Figure 5. The exemplary FN-curve used for calculate societal risk.

The Variants including a sprinkler system or mechanical ventilation are characterized by a fairly low level of societal risk. This is especially evident in the risk profiles for deaths. For these variants, the maximum number of people who may be injured is lower than in other variants. In addition, risk profiles are characterised by a large inclination indicating a significant reduction in the likelihood of injury along with the number of people at risk.

Table 2. A summary of risk values for the presented solutions. R1 - evacuees will inhale a negligible amount of fire gases; R2 - evacuees will inhale an amount of fire gases resulting in minor injury. R3 evacuees will inhale an amount of fire gases resulting in severe injury. R4 - evacuees will inhale an amount of fire gases resulting in death.

\begin{tabular}{|l|c|c|c|c|c|c|}
\hline Risk & V1.1 & V1.2 & V2.1 & V2.2 & V2.3 & V2.4 \\
\hline R1 & $2.28 \times 10^{-6}$ & 0 & $2.38 \times 10^{-6}$ & $1.80 \times 10^{-5}$ & 0 & 0 \\
\hline R2 & $1.62 \times 10^{-5}$ & $1.47 \times 10^{-5}$ & $3.73 \times 10^{-5}$ & $7.69 \times 10^{-5}$ & $1.84 \times 10^{-5}$ & $3.65 \times 10^{-5}$ \\
\hline R3 & $1.40 \times 10^{-5}$ & $1.82 \times 10^{-6}$ & $4.59 \times 10^{-6}$ & $3.40 \times 10^{-5}$ & $1.16 \times 10^{-5}$ & $2.31 \times 10^{-5}$ \\
\hline R4 & $1.26 \times 10^{-4}$ & $1.82 \times 10^{-6}$ & $1.16 \times 10^{-5}$ & $1.36 \times 10^{-5}$ & $1.21 \times 10^{-4}$ & $1.42 \times 10^{-4}$ \\
\hline
\end{tabular}

\section{Discussion}

When assessing the level of fire safety, it was found that the safest option was variant 1.2, which involved smoke ventilation in the corridors and mechanical ventilation. Next was variant 2.1 with mechanical ventilation, 2.2 with the sprinkler system, 2.3 with procedures, 1.1 involving interior re-arrangement and finally 2.4 with illuminated emergency exit signs. 
Table 3 presents the ranking of the fire protection concept variants according to the $\mathrm{R} 4$ risk level (evacuees inhaling an amount of fire gases sufficient to lead to death).

Table 2 shows an interesting regularity. There are very large discrepancies in the risk values for solutions in the same category: direct adjustment to the requirements defined by the regulations. Variant 1.2 opens the table with the lowest risk, while option 1.1 closes it as the penultimate one.

However, it should be noted that option 1.1, as a variant complying with the regulations, set the acceptable level of fire risk. Thus, each of the options analysed in this case study would ensure a level of security that was not worse than the one required by law, which is a convincing argument for accepting these solutions as alternative solutions.

When assessing the fire safety level by means of multisimulation, the existing architectural layout, the specified number of people and the building's fire protection devices and installation equipment were all taken into account, including the scenario of their joint action and basic design parameters. As a result, a risk value was obtained, which, by taking into account a large number of input variables, reflected the actual risk from among all known and popular methods of analysis. Unfortunately, by striving to reproduce reality, it limited the possibility of drawing general conclusions. Therefore, the authors consider that option 2.3, with procedures and audits, provides a level of fire safety that is not worse than that specified in the regulations only in this particular case, and that it is not appropriate to approximate the conclusion to seemingly similar cases.

\section{References}

[1] A. Krasuski, P. Kubica, M. Pecio, Ochr. Ppoz 60 (2017)

[2] N. Metropolis, S. Ulam, J. Am. Stat. Assoc. 44, 335 (1949)

[3] National Fire Protection Association, NFPA 551-Guide for the Evaluation of Fire Risk Assessments (2007)

[4] National Fire Protection Association, NFPA 101 life safety code (2011)

[5] International Organization for Standardization, Fire safety engineering - Fire risk assessment - Part 1: General (2012), ISO 16732-1:2012(E)

[6] National Fire Protection Association, SFPE engineering guide to performance-based fire protection: analysis and design of buildings (NFPA, 2000)

[7] Hurley, M. J. et. al., SFPE handbook of fire protection engineering (Springer, 2015)

[8] BSI, Part 7: Probabilistic risk assessment, in PD 7974-7:2004, The application of fire safety engineering principles to fire safety design of buildings (British Standard, 2003)

[9] S. Hostikka, O. Keski-Rahkonen, Nucl. Eng. Des. 224, 301 (2003)

[10] C. Wade, G. Baker, K. Frank, R. Harrison, M. Spearpoint, B-RISK 2016 user guide and technical manual. Study Report SR364, BRANZ (2016)

[11] A.A. Stec, T.R. Hull, Fire toxicity (Elsevier, 2010)

[12] R.W. Bukowski, F. Clarke, J.R. Hall, S. Stiefel, Fire risk assessment method: description of methodology (Center for Fire Research, 1990)

[13] L.A. Cox Jr, Risk analysis foundations, models, and methods, Vol. 45 (Springer Science \& Business Media, 2012) 